\title{
Antipsychotic prescribing in light of the consensus statement of the College
}

\author{
K. L. Newton, R. Murthy and J. Qureshi
}

\begin{abstract}
A retrospective survey was undertaken to evaluate the prescribing practices of medical stafl in a Distict General Hosplial in IIght of the Consensus Statement by the Royal College of Prychiatists on the use of high dose antipsychotics (1993). Two per cent of 247 patients were prescribed a higher than recommended dose of antipeychofic. None of these cases had been prescribed 'supra-BNF doses of antipsychotic on a regular basis, but with the addition of 'cis required' (PRN) medication the recommended dose was exceeded. In onty one patient was the high doscoge administered. A further analysis of these patients is made.
\end{abstract}

The relationship between dose and therapeutic efficacy of antipsychotics prescribed for patients suffering with schizophrenia remains unclear. It has been the rationale that very high doses may be required in a few patients to block dopamine receptors satisfactorily. Controlled studies comparing standard with high doses in treament resistant' patients have failed to show superior effectiveness of megadose regimes (Bjorndal et al. 1980; Kane, 1987). These findings are supported by positron emmission tomography studies which have shown that a level of dopamine D2 occupancy associated with antipsychotic efficacy $(>65 \%)$ occurs at modest doses, e.g. chlorpromazine 300-400 mg (Farde et al, 1992). Patients who were resistant to normal doses of antipsychotics still had 80 $85 \%$ of receptors occupied and were indistinguishable from those who responded to such drugs (Wolkin et al, 1989).

For nearly all antipsychotic drugs the $B N F$ recommends a maximum dose, above which the risk of side-effects becomes unacceptable to the risk-benefit ratio. An agreed definition of high dose is "a total daily dose which exceeds the advisory upper limit for general use in the BNF product licence" (Royal College of Psychiatrists, 1993). There have been publications advising against the use of high dose of antipsychotics except as a last resort (Baldessarini et al, 1988; Drug and Therapeutics Bulletin, 1992).

A recent audit on the use of antipsychotic medication in a large psychiatric hospital found that nearly half of the patients on antipsychotics were prescribed doses in chlorpromazine equivalents in excess of BNF guidelines (Warner et al, 1995). However, this hospital included a regional unit for patients suffering with treatment-resistant schizophrenia. The sample may therefore have included a higher than average proportion of patients prescribed high dosages of antipsychotics. More recently Krasucki \& McFarlane (1996) reported a rate of high-dose antipsychotic prescription of just $7.3 \%$ among a cross-section of all psychiatric in-patients, a rate equivalent to $14.1 \%$ of those patients prescribed antipsychotics. This figure rose to $42.4 \%$, however, when what might have been given within the confines of the prescription chart were considered (our calculations from Krasuck's data).

This study was designed to determine whether patients in a district general hospital are regularly prescribed antipsychotic medication in doses that exceed those recommended by the BNF.

\section{The study}

New Cross Hospital is a district general hospital serving a catchment population of 250000 . A retrospective analysis was made of all schizophrenic patients receiving in-patient care during 1993. The age, gender, clinical diagnosis, and details of all prescribed medication during the inpatient period were available for every patient from the case notes and prescription charts. Multiple admissions during this study period were considered together as a single 'in-patient' contact for each individual. The total dose for 
each antipsychotic was converted into chlorpromazine equivalents using the $B N F$ conversion table. Those patients prescribed antipsychotics in excess of the maximum BNF dosage recommendations were identified and studied further. In these cases the following were noted: (1) patient ethnicity; (2) the seniority of the prescribing physician; (3) the rationale for the prescription of high-dose antipsychotics (using the case notes) and, (4) whether the prescribed total daily dose was actually dispensed.

\section{Findings}

The case notes and medication charts of 247 patients were examined. We found that only five (2\%) in-patients had been prescribed antipsychotics in doses exceeding those recommended by the $B N F$. These five patients were male; four were Afro-Caribbean. In each of these five cases the prescription of regular medication was within dosage guidelines, but with the addition of as required' (PRN) drugs the total recommended dose of antipsychotics had been exceeded. In only one case, however, was the high dose actually administered. No documentation was made in any of the case notes to explain the potentially excessive combined dosage, and junior medical staff had made all but one of these prescriptions.

\section{Comment}

This study indicates that very few patients were prescribed 'supra-BNF' doses of antipsychotic. Our findings are contrary to those of Warner et al (1995) who demonstrated over-prescribing in nearly half of schizophrenic cases. However, their study was conducted among a sample including treatment-resistant schizophrenic patients which may account for the differences found. The rate of prescription of high dose antipsychotics in Krasucki's study was only $14.1 \%$, but of more concern were the $42.4 \%$ of patients who potentially could receive high doses as a combination of regular plus as required (PRN) medication.

All our patients had been prescribed antipsychotics within BNF guidelines for regular dosing, but any addition of PRN medication would have exceeded the recommended limit. Krasucki \& McFarlane (1996) raised a valuable point that some patients are likely to have been written up for these potentially large antipsychotic doses inadvertently, as chlorpromazine dose equivalent totals take time to calculate and require conversion data. The decision to administer these excessive quantities if and when needed was effectively passed to nursing staff.

Many of the PRN prescriptions were designated 'for oral or intramuscular use'. As the blood level of a drug administered by parenteral injection may reach up to five times the drug level achieved by oral administration, the combined total dose may have achieved significantly greater blood levels.

The Consensus Statement (Royal College of Psychiatrists, 1993) suggests that the decision to prescribe high-dose antipsychotic medication should be undertaken only by a senior psychiatrist, and the reasons should be stated clearly in the case notes, a practice not adhered to in our sample. It is possible, however, that the prescriptions made by junior staff were authorised by a senior psychiatrist quite legitimately. but documentation within the case notes was not made.

Four of the five patients prescribed high doses were Afro-Caribbean males. These patient numbers are of course very small, and we are unaware of any previous documention of potential differences in either prescribing practice, or drug response observed between patients of different ethnic origins.

In summary, the proportion of patients prescribed high-dose antipsychotic medication was very small among a population of in-patients with schizophrenia. BNF recommended doses were not exceeded by regular prescription, but clinicians should be alert to the potential cumulative dose implications of concurrent as required' medication.

\section{References}

Baldessarini, R. J., Cohen, B. M. \& TRICher, M. H. (1988) Significance of neuroleptic dose and plasma level in the pharmacological treatment of psychoses. Archives of General Psychiatry. 46, 79-89.

BJORNDAL, E., BJEERE, M.. GERLACH, J., et al (1980) High dose haloperidol therapy in chronic schizophrenic patients. A double blind study of clinical response. side-effects, serum haloperidol and serum prolactin. Psychopharmacology, 67, 17-23.

FARDE, L., NONDSTRON, A. L., WIESEL, F. A., et al (1992) Positron emmision tomographic analysis of central Dl and D2 dopamine receptor occupancy in patients treated with classical neuroleptics and clozapine. Archives of General Psychiatry. 49, 538-544.

KANE, J. M. (1987) Treatment of schizophrenia. Schizophrenia Bulletin, 13, 133-156.

KRASUCKI, C. \& MCFARLANE, F. (1996) Electrocardiograms, high-dose antipsychotic treatment and College guidelines. Psychiatric Bulletin, 20, 326-330.

ROYAL COLLEGE OF PSYCHIATRISTS (1993) Consensus Statement on the Use of High Dose Antipsychotic Medication. London: RCPsych.

WARNER, J. P., SLADE, R. \& BARNES, T. R. E. (1995) Change in neuroleptic prescribing practice. Psychiatric Bulletin. 19. 237-239. 
Wolkin, A. Barouche, F., Wolfe, A. P., et al (1989) Dopamine receptor blockade and clinical response; evidence for two biological subgroups of schizophrenia. American Journal of Psychiatry, 146. 905-908.

Prescribing for unlicensed drugs or using drugs for unlicensed indications. Drug and Therapeutics Bulletin (1992) 30. 97-99.
${ }^{*} \mathrm{~K}$. L. Newton, South Kensington and Chelsea Mental Health Centre, 1 Nightingale Place, London, SW10 9NG; R. Murthy and J. Qureshi, New Cross Hospital, Wolverhampton WV10 OQP

*Correspondence

\title{
Psychological Trauma - A Developmental Approach
}

\author{
Edited by Dora Black, Martin Newman, Jean Harris Hendriks and Gillian Mezey
}

This is the first UK textbook on psychological trauma and contains contributions by many of the country's leading authorities on responses to traumatic events. It is edited by four clinicians with extensive experience on this subject.

The book discusses normal and abnormal responses to stress, disasters, war and civil conflict, and interpersonal violence, diagnosis, interventions and treatments, and legal aspects. There is reference throughout to the research findings, and discussion of future research needs. Each chapter contains a comprehensive bibliography for those who wish to read further. Published January 1997, price £30.00, 424pp. ISBN 0902241982

Gaskell is the imprint of the Royal College of Psychiatrists. Gaskell books are available from good bookshops and from the Publications Department, Royal College of Psychiatrists, 17 Belgrave Square, London SW1X 8PG (Tel. +44(0)171 235 2351, extension 146). The latest information on College publications is available on the INTERNET at: http://www.demon.co.uk/rcpsych/

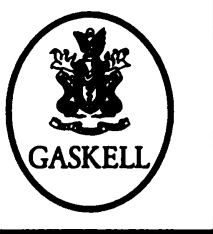

